\title{
Constraints on the Polarization of the Anomalous Microwave Emission in the Perseus Molecular Complex from 7-year WMAP data
}

\author{
C. H. López-Caraballo, J. A. Rubiño-Martín, R. Rebolo, R. Génova-Santos \\ clopez@iac.es, jalberto@iac.es, rrl@iac.es, rgs@iac.es \\ Instituto de Astrofísica de Canarias (IAC), C/Vía Láctea, s/n, E-38200, La Laguna, Tenerife (Spain)
}

\begin{abstract}
We have used the seven year Wilkinson Microwave Anisotropy Probe (WMAP) data in order to update the measurements of the intensity signal in the G159.6-18.5 region within the Perseus Molecular Complex, and to set constraints on the polarization level of the anomalous microwave emission in the frequency range where this emission is dominant. At 23,33 and $41 \mathrm{GHz}$, we obtain upper limits on the fractional linear polarization of 1.0, 1.8 and $2.7 \%$ respectively (with a 95 per cent confidence level). These measurements rule out a significant number of models based on magnetic dipole emission of grains that consist of a simple domain (Draine \& Lazarian 1999) as responsible of the anomalous emission. When combining our results with the measurement obtained with the COSMOSOMAS experiment at $11 \mathrm{GHz}$ (Battistelli et al. 2006), we find consistency with the predictions of the electric dipole and resonance relaxation theory (Lazarian \& Draine 2000) at this frequency range.
\end{abstract}

Subject headings: diffuse radiation - radiation mechanisms: general - radio continuum:ISM ISM:individual(G159.6-18.5) - cosmic microwave background

\section{Introduction}

The dust-correlated microwave $(10-60 \mathrm{GHz})$ emission detected by several cosmic microwave background $(\mathrm{CMB})$ experiments performed in the two last decades (COBE (Kogut \& Hinshaw 1996); OVRO at 14.5 and $32 \mathrm{GHz}$ (Leitch et al. 1997); Saskatoon (de Oliveira-Costa et al.1997); $19 \mathrm{GHz}$ (de Oliveira-Costa et a 1998); Tenerife (de Oliveira-Costa et al. 1999, 2004); COSMOSOMAS (Watson et al. 2005; Hildebrandt et al. 2007); VSA (Scaife et al. 2007; Tibbs et al. 2010); and references therein) suggests the existence of a new continuum microwave emission mechanism unlike to the three well-known Galactic mechanisms: synchrotron, free-free and thermal dust emission. A remarkable observational effort has been devoted to the understanding of the intensity and polarization properties of this "anomalous" microwave emission, among other reasons, because of the importance of an accurate foreground correction of the $\mathrm{CMB}$ maps at low frequencies.

Among the various scenarios proposed to explain this emission, electric dipole radiation (Draine \& Lazarian
1998) from very small $\left(\mathrm{N} \leq 10^{3}\right.$ atoms) rapidly rotating $\left(\sim 1.5 \times 10^{10} \mathrm{~s}^{-1}\right)$ carbon based molecules in the interstellar medium (the so-called "spinning dust") appears to reproduce well the observational constraints (see e.g. Watson et al. 2005; Casassus et al. 2006; Iglesias-Groth 2006; Dickinson et al. 2007; Tibbs et al. 2010). The detailed theoretical description .of this family of models has been recently updated in Ali-Haïmoud et al. (2009), also including the effect of rotation of the dust grains around a non-principal axis (Silsbee et al. 2010).

An alternative explanation based on magnetic dipole emission of spinning dust has also been proposed (Draine \& Lazarian 1999). Measurements of the polarization properties of the anomalous microwave emission may potentially distinguish between these two models. According to Lazarian \& Draine (2000), electric dipole radiation from spinning dust would be polarized at low frequencies, reaching a maximum (6-7\%) at $2-3 \mathrm{GHz}$ and dropping to $4-5 \%$ at $10 \mathrm{GHz}$ and progressively decreasing at higher frequencies. Polarization from magnetic dipole emission predicts a different frequency behaviour and stronger 
linear polarization depending on the composition and shape of the emitting particles.

From the observational point of view, there is little information in the literature about the polarization properties of the anomalous emission. Kogut et al. (2007) used the full-sky WMAP 3-year data to constrain the polarization fraction of a diffuse anomalous component traced by the dust morphology. They concluded that the polarized anomalous emission contributes less than $1 \%$ of the observed polarization signal variance in any of the five WMAP bands.

Only few attempts have been made to determine the polarization of the anomalous microwave emission in individual objects, and in the frequency range where this contribution is dominant $(10-50 \mathrm{GHz})$. Battistelli et al. (2006), using data from the COSMOSOMAS experiment on the Perseus molecular complex, reported at $11 \mathrm{GHz} \Pi=3.4_{-1.0}^{+1.5} \%$ (95\% confidence level). More recently Mason et al. (2009), using the Green Bank Telescope at $9 \mathrm{GHz}$, obtained an upper limit of linear polarization of $88 \mu \mathrm{K}$ at $95.4 \%$ of confidence in the Lynds 1622 dark cloud. Both the Perseus molecular complex and the Lynds 1622 cloud are regions where anomalous microwave emission appears to dominate on other emission processes in the frequency range 10-50 GHz.

In this work, we present new measurements of the polarization of the anomalous microwave emission in G159.6-18.5 (within the Perseus molecular complex) using the WMAP (Wilkinson Microwave Anisotropy Probe) 7-year data. Although the results only provide upper limits to the polarized emission in the region, they still constitute a strong constraint on the physical mechanism responsible for the emission.

\section{The G159.6-18.5 region}

The Perseus molecular complex is a giant molecular cloud located at a distance of $260 \mathrm{pc}$ (Cernicharo et al. 1985). Our region of interest is G159.6-18.5 , a dust feature in this molecular complex, observed in the IRIS1 maps, which appears as a slightly broken ring with a diameter $\approx 1.5^{\circ}$, and a intensity of the order of 100-200 $\mathrm{MJy} \mathrm{sr}^{-1}$ at $100 \mu \mathrm{m}$ and 5-10 $\mathrm{MJy} \mathrm{sr}^{-1}$ at $12 \mu \mathrm{m}$ (see Figure 1).

Originally, G159.6-18.5 was considered to be a supernovae remnant Pauls \& Schwartz 1989; Fiedler et al.

${ }^{1}$ IRIS are improved version of IRAS maps, for details see Miville-Deschênes \& Lagache (2005).
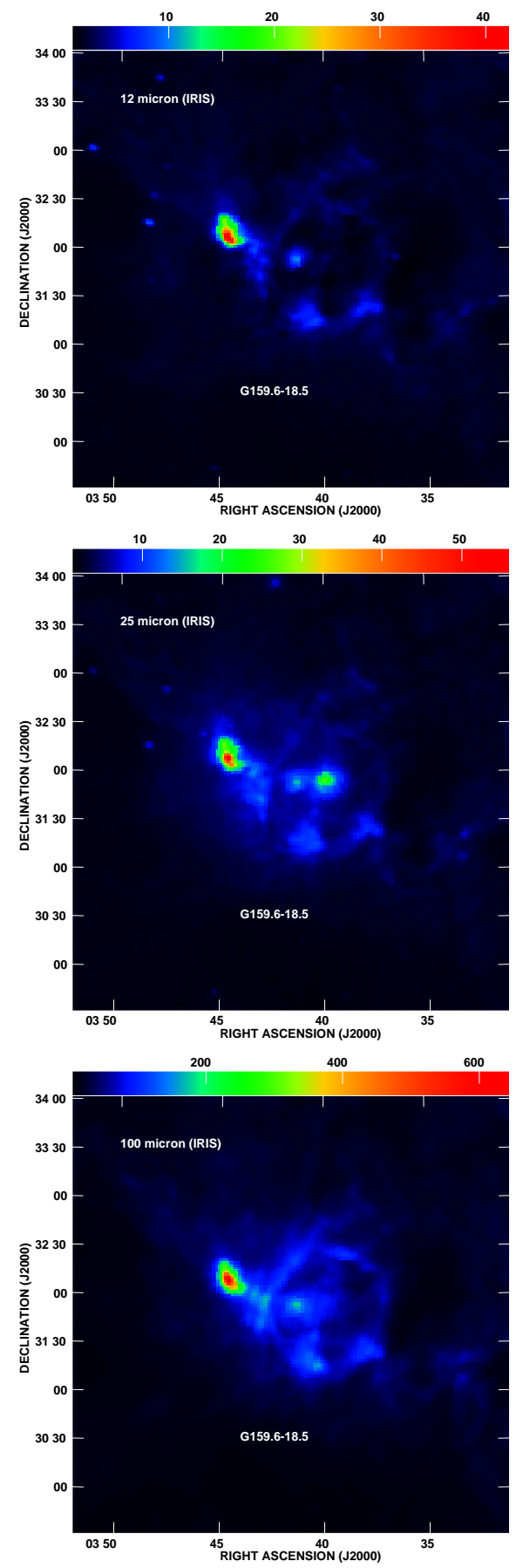

Fig. 1.- : IRIS images at $12 \mu \mathrm{m}, 60 \mu \mathrm{m}$ (middle) and $100 \mu \mathrm{m}$ (bottom panel) of the G159.6-18.5 region, with a field of view of $4.5^{\circ} \times 4.5^{\circ}$, and centered in R.A. (J2000) $=55.4^{\circ}$ and Dec. $=+31.8^{\circ}$. All maps are in units of $\mathrm{MJy} \mathrm{sr}^{-1}$. 
1994), but later observations showed that this is a $\mathrm{H}_{\text {II }}$ region driven by the O9.5-B0 V star HD 278942 , located at the center of the ring (de Zeeuw et al. 1999). More recent studies (Andersson et al. 2000; Ridge et al. 2006)) concluded that G159.6-18.5 was indeed an expanding $\mathrm{H}$ in bubble that has emerged from the outer edge of the cloud.

Watson et al. (2005, hereafter W05) carried out a detailed study of the spectral energy distribution (SED) of G159.6-18.5 in a wide frequency range, from $408 \mathrm{MHz}$ to $3000 \mathrm{GHz}$. This study combined, among others, the observations performed with the COSMOSOMAS experiment at 11,13,15 and $17 \mathrm{GHz}$ Gallegos et al. 2001; Fernández-Cerezo et al. 2006), and the 3rd year WMAP data (Bennett et al. 2003). The total emitting region in G159.6-18.5 was slightly resolved in COSMOSOMAS and WMAP data, and was modelled using an ellipse with $1.6^{\circ} \times 1.0^{\circ}$ and P.A. $=51^{\circ}$, centered on R.A. $=55.4^{\circ}$ and Dec. $=$ $+31.8^{\circ}$. The SED for that region showed clear evidence for anomalous microwave emission, with a peaked spectrum around $20-30 \mathrm{GHz}$, indicative of spinning dust. Indeed, W05 showed that an adequate fit to the SED can be achieved from 10 to $50 \mathrm{GHz}$ only when including three components in the analysis: (a) optically thin free-free emission; (b) vibrational dust emission with $T_{\text {dust }}=19 \mathrm{~K}$ and emissivity index of 1.55; and (c) a combination of the spinning dust models of Draine \& Lazarian (1998) for warm neutral medium and molecular cloud $(0.8 \mathrm{WNM}+0.3 \mathrm{MC})$. No bright unresolved source that could be ultra-compact $\mathrm{H}$ II region or gigahertz-peaked source could be found.

Detailed observations of G159.6-18.5 with the Very Small Array (VSA) interferometer at $33 \mathrm{GHz}$ (Tibbs et al. 2010) and an angular resolution of 1040 arcmin, showed that the region consists of five distinct components, all of which are found to exhibit a emission at $33 \mathrm{GHz}$ which is highly correlated with the far-infrared emission. The most interesting result is that the VSA resolved out most of the emission in the region, as those five components contribute to only $\approx 10$ per cent to the total flux density of the diffuse extended emission detected in W05. Therefore, the bulk of the anomalous emission in G159.6-18.5 is diffuse.

Concerning the polarization level of the anomalous microwave emission in the region, the only measurement at the relevant frequencies $(10-30 \mathrm{GHz})$ done so far was presented in Battistelli et al. (2006). Using dual orthogonal polarizations with the COSMOSOMAS experiment, the resulting total polarization level in G159.6-18.5 at $11 \mathrm{GHz}$ was found to be $\Pi=$ $3.4_{-1.9}^{+1.5} \%$ (95\% confidence level). Based on this value, they concluded that this weak detection of polarization would be associated to the spinning dust grains. Recently, Reich \& Reich (2009) suggested that G159.618.5 is acting as a Faraday screen hosting a strong regular magnetic field, which would rotate the background polarized emission. They suggest that a Faraday screen model with a rotation measure of $R M=$ $190 \mathrm{rad} \mathrm{m}^{-2}$ would explain the $11 \mathrm{GHz}$ observations, as well as their new $11 \mathrm{~cm}$ Effelsberg observations in the region (see Fig. 6 in that paper). In any case, even if the Battistelli et al. (2006) result is only an upper limit to the polarization level of the anomalous emission, its amplitude favours the electric dipole emission model with resonance paramagnetic relaxation (see Lazarian \& Draine (2000)).

In this context, the seven-year WMAP data represent an opportunity to update the measurements of intensity signal in the Perseus region, as well as to constrain the polarization level of the anomalous microwave emission in the frequency range where this emission is dominant $(20-30 \mathrm{GHz})$. As shown by Tibbs et al. (2010), 90\% of the emission in the region is diffuse, so the angular resolution of WMAP data is sufficient to provide a reliable measurement.

\section{Data and Methodology}

\subsection{WMAP data}

In this paper, we use the seven-year WMAP data products (Jarosik et al. 2010), which are available in the LAMBDA $\sqrt{2}$ website in the HEALPIX] 3 pixelisation scheme (Górski et al. 2005). In particular, we use the smoothed I, Q and U maps, for each of the five frequency bands centered at 23, 33, 41, 61 and $94 \mathrm{GHz}$ ( $\mathrm{K}, \mathrm{Ka}, \mathrm{Q}, \mathrm{V}$ and $\mathrm{W}$ respectively). The original angular resolution of these maps is approximately 0.82 , $0.62,0.49,0.33$, and $0.21 \mathrm{deg}$ respectively, but they are degraded to a common resolution of $1 \mathrm{deg}$. For each map, a full description of the noise covariances (II, QQ, UU, QU) is provided. This information will be used in our analyses. We would like to stress that the WMAP polarization maps are derived from the difference of two differential measurements. Thus, the WMAP measures a double difference in polarized intensity, not the intensity of the difference of the electric

\footnotetext{
${ }^{2}$ http://lambda.gsfc.nasa.gov/

${ }^{3}$ http://healpix.jpl.nasa.gov
} 

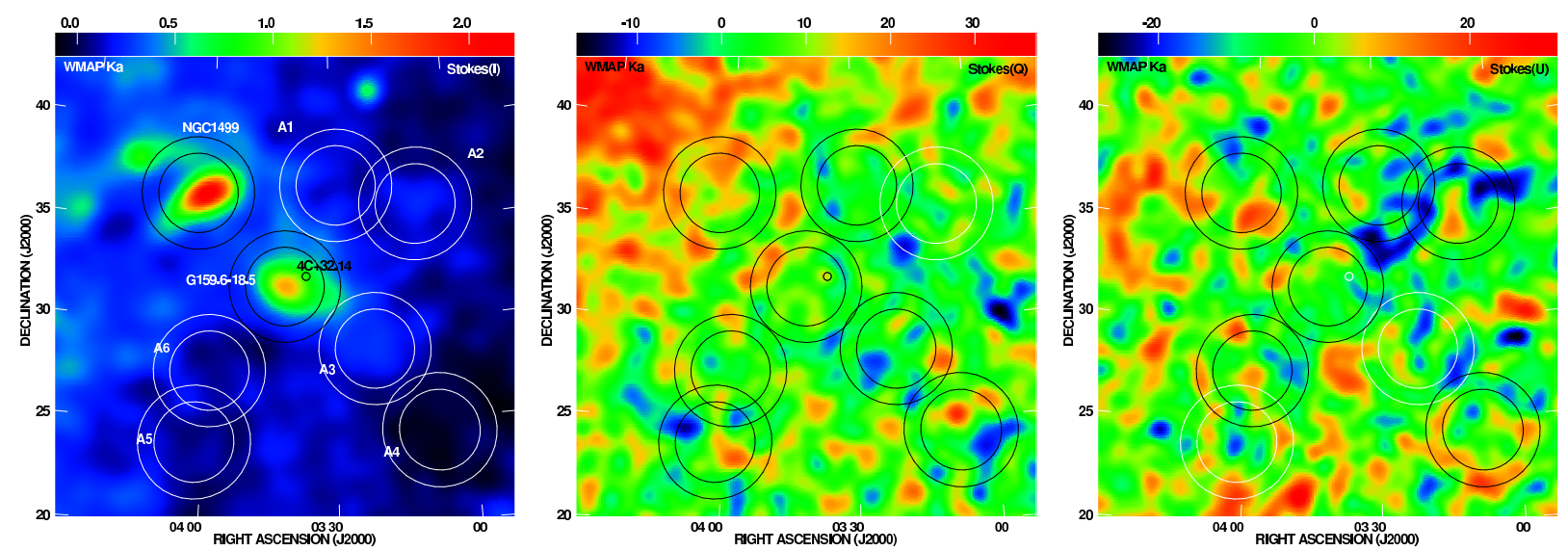

Fig. 2.-: Neighborhood of the Perseus Molecular Complex as seen by WMAP-7 at $33 \mathrm{GHz}$ (Ka band), with field of view of $23^{\circ} \times 23^{\circ}$ centered in the G159.6-18.5 region. All images (I, right panel; Q, center; and U, left panel) are smoothed to $1^{\circ}$ resolution, and in units of $\mathrm{mK}$ (thermodynamic). The black circles define the circular apertures around the objects studied in this paper (see text for details).

field as with interferometers and correlation receivers (for further details, see Page et al. 2007; Jarosik et al. 2007; Kogut et al. 2003).

From those $\mathrm{Q}$ and $\mathrm{U}$ maps at each frequency band, we can obtain the maps of the polarization intensity as $P=\sqrt{Q^{2}+U^{2}}$, while the angle between the polarization direction of the electric field and the Galactic meridian can be obtained as $\gamma=\frac{1}{2} \arctan (U / Q)$.

Figure 2 shows a detailed view of the Perseus Molecular Complex as seen in the Ka-band of WMAP $(33 \mathrm{GHz})$. In this map, two strong sources are clearly visible: G159.6-18.5 (placed at the center of the image), and the $\mathrm{H}_{\text {II }}$ region NGC1499. This later object will be used as null hypothesis in our study, as free-free emission is known to be unpolarized. This null-test was also used in the study of Battistelli et al. (2006). Finally, the figure also displays six nearby regions which have been selected due to their low emission in intensity. These regions will be used below to characterize the behaviour of the background diffuse emission in the surroundings of G159.6-18.5 .

\subsection{Ancillary data}

\subsubsection{Intensity}

As a reference for the SED in G159.6-18.5, in this work we use the data points from Table 1 in W05. In that table, in addition to the COSMOSOMAS and WMAP measurements, there are also data points at $0.408 \mathrm{MHz}$ (Haslam et al. 1982); at $1420 \mathrm{MHz}$
(Reich \& Reich 1988); and at 100, 140 and $240 \mu \mathrm{m}$, based on the DIRBE maps (Silverberg et al. 1993).

Here we have re-evaluated the intensity measurements on the Haslam, WMAP and DIRBE maps, using the methodology described in Section 3.3 The derived fluxes are slightly higher than those presented in W05 (by a factor of 5-13 per cent), although as pointed out below, this is probably due to the different methodology used for the flux determination in W05.

After W05, Semenova et al. (2009) used the RATAN600 telescope to obtain independent measurements in the region. Although an estimate of the error bar of these new measurements is not provided in their paper, the overall shape of the SED in G159.6-18.5 in the frequency range $1-20 \mathrm{GHz}$ is fully compatible with the results of W05, showing a rising spectrum in that frequency range.

Recently, Reich \& Reich (2009) presented preliminary results of the emission in G159.6-18.5 at $11 \mathrm{~cm}$ and $6 \mathrm{~cm}$ with the Effelsberg and the Urumqi telescopes, respectively. These results support that the emission in the frequency range $2.7-5 \mathrm{GHz}$ is compatible with an optically thin thermal gas, as expected from the SED in W05.

\subsubsection{Polarization}

The only reported measurement to date of the radio polarized intensity in G159.6-18.5 comes from the COSMOSOMAS experiment at $11 \mathrm{GHz}$ (Battistelli et al. 2006). The measured fractional polarization is $\Pi=$ 

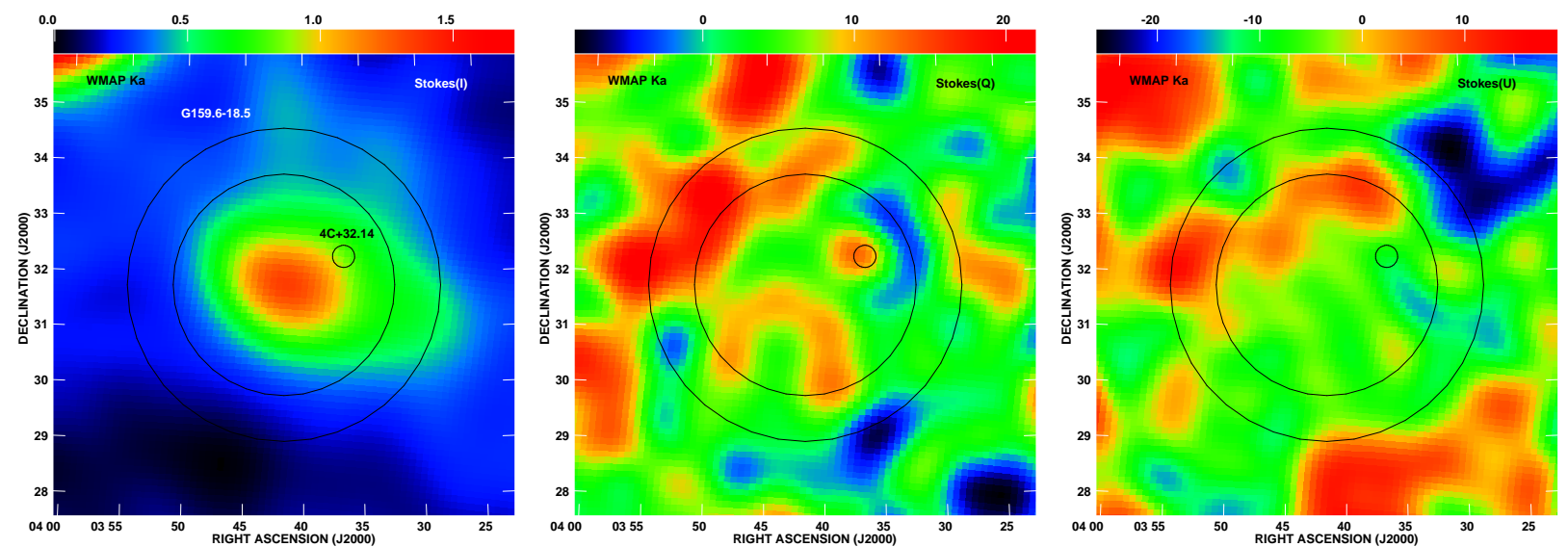

Fig. 3.- : Close view of G159.6-18.5 as seen by WMAP-7 at $33 \mathrm{GHz}$ (Ka band), with a field of view of approximately $8^{\circ} \times 8^{\circ}$. The images show I (right frame), Q (central frame) and $\mathrm{U}$ (left frame) at $1^{\circ}$ resolution, and in units of $\mathrm{mK}$ (thermodynamic). The small aperture at the northwest of the center of G159.6-18.5 corresponds to the solid angle defining the quasar $4 \mathrm{C}+32.14$. The pixels within this small aperture were not considered in our flux estimation.

\section{$3.4_{-1.9}^{+1.5 \%}$ (95\% C.L.).}

Recently, Reich \& Reich (2009) presented preliminary results of the polarized emission in G159.618.5 at $11 \mathrm{~cm}(\sim 2.7 \mathrm{GHz})$ with the Effelsberg telescope. They reported a significant degree of polarization along the ring, which they interpreted as an indication that G159.6-18.5 acts as a Faraday screen rotating the polarization angle of the background emission in the region. However, this effect will be strongly suppressed at the peak frequencies of the anomalous emission $(20-30 \mathrm{GHz})$ as discussed below.

Kogut et al. (2007) presented a full-sky model of the polarized Galactic microwave emission, considering in their analysis the polarization from the thermal dust and synchrotron emissions. The resulting polarized dust model obtained in this paper shows that the Perseus molecular complex lies near to the boundaries of the P06 mask (see mask definition in Page et al. 2007), where the expected dust fractional polarization $\left(\Pi_{\text {dust }}\right)$ reaches $6.4 \pm 1.3 \%$ and $2.0 \pm 1.4 \%$ for $|b|<10 \mathrm{deg}$ and $|b|>10 \mathrm{deg}$, respectively, inside P06; and $3.6 \pm 1.1 \%$ outside the P06 mask. This fractional degree of polarization will be of importance when studying the high frequency behaviour of the polarized emission in the G159.6-18.5 region.

\subsection{Determination of the Spectral Energy Distri- bution in intensity and polarization}

Figures 2 and 3 show that there is no obvious structure seen within the G159.6-18.5 region in the polar- ization maps. In this case, we can not apply a similar method to the one used in W05 for the flux determination. In that paper, a simple elliptical Gaussian model was used to describe the region, and fluxes were obtained using a direct fitting to the data.

Here, we will adopt a direct aperture integration, or "ring analysis", both for the intensity and polarization determinations. The practical implementation of this method has been previously used in other works (Bennett et al. 1993; Banday et al. 1996; Hernández-Monteagudo \& Rubiño-Martín 2004). The basic idea is as follows. For a certain frequency map, we define a circular aperture around the object with radius $r_{1}$, defining a solid angle $\Omega_{\mathrm{S}}$ around the source. In order to correct for the background emission, a circular corona with radius $r_{1}<r<r_{2}$ is used. In order to preserve a similar area both for the source and the background flux estimation, we use the relation $r_{2}=r_{1} \sqrt{2}$ between the two apertures. This method provides an efficient correction of the background emission around the source for apertures ( $r_{1}$ values) comparable to the beam size, although for larger apertures might be biased due to the background or CMB contributions. The error bar associated to this measurement contains the quadratic sum of two contributions, the pure instrumental noise and the increase of the variance due to the CMB fluctuations. It is important to note that the evaluation of both contributions should account for correlation terms. In the case of the CMB, this is introduced by means of the two-point correlation func- 
tion, which is evaluated using the (measured) WMAP angular power spectrum. In the case of the noise, the correlation between pixels appears after the smoothing process, and is taken into account in a pixel-by-pixel basis.

As we will see below, in the case of the selected aperture for G159.6-18.5 $\left(r_{1}=2^{\circ}\right)$ and the instrumental noise level of the WMAP data, the CMB contribution to the error bar is dominant for the intensity Stokes parameter, while its contribution is negligible for Stokes Q and U. Although the main focus of this paper is the polarization determination, for illustration we have also considered how the intensity measurements change with our aperture method when a CMB correction is performed before the flux determination. For that discussion, we have used the Internal Linear Combination (ILC) map provided by the WMAP team in the LAMBDA webpage (Gold et al. 2010). Although this map does not provide an optimal separation of CMB component, it provides a reasonable approximation which can be used to estimate its impact on the final intensity measurements.

\section{Results and Discussion}

\subsection{Control regions}

\subsubsection{Characterization of the background emission}

G159.6-18.5 is relatively close to the galactic plane, in a region with a non-negligible diffuse emission which varies markedly with the position. In order to characterize the behaviour of this background emission, we have selected six regions with low emission in intensity in the surroundings of the Perseus molecular complex. The central coordinates of the six regions are shown in table 1 Around each of them, we have chosen the same aperture as for G159.6-18.5 $\left(r_{1}=2^{\circ}\right)$ for the flux determination.

Table 2 summarizes our results. In addition to the I, $\mathrm{Q}$, and $\mathrm{U}$ determinations at each frequency, we also include in the last two columns the values for the fluxes

Table 1:: Identification number and coordinates of the six control regions in the surroundings of G159.6-18.5 used for the background characterization. The aperture used in all cases and at all frequencies is $r_{1}=2^{\circ}$.

\begin{tabular}{ccccccc} 
ID & A1 & A2 & A3 & A4 & A5 & A6 \\
\hline \hline R.A. $\left[{ }^{\circ}\right]$ & 52.19 & 47.30 & 50.21 & 46.88 & 60.39 & 59.70 \\
DEC. $\left[{ }^{\circ}\right]$ & 36.88 & 35.74 & 28.56 & 24.34 & 23.88 & 27.48 \\
\hline
\end{tabular}

inside the inner aperture $\left(r_{1}\right)$ with no background correction. These numbers, quoted as $\mathrm{Q}_{\text {back }}$ and $\mathrm{U}_{\text {back }}$, will be used below for the analysis of the Faraday rotation in the region (Sect. 4.2). The table also includes an estimation of the total linear polarization $\mathrm{P}=\sqrt{\mathrm{Q}^{2}+\mathrm{U}^{2}}$, and also the upper limit on this linear polarization $\mathrm{P}_{0}$ that we would obtain in the nullhypothesis case using a zero signal $(\mathrm{Q}=\mathrm{U}=0)$ and the same noise levels. As in all these values no detection is found, we quote upper limits which correspond to the $95 \%$ confidence level derived from a maximum likelihood approach based on the $\mathrm{Q}$ and $\mathrm{U}$ measurements.

We first note that at all frequencies, the main contribution to the error budget in intensity comes from the CMB part, which for illustration purposes is written separately in the table 2 (values in brackets in the second column). As one would expect, its relative contribution rises with approximately $v^{2}$ dependence, as the temperature sensitivity of WMAP is comparable in all channels. In the case of $\mathrm{Q}$ and $\mathrm{U}$, the $\mathrm{CMB}$ contribution is negligible and it is not included in the error bar.

Finally, as a summary of the results in table 2, we present in table 3 the peak-to-peak variations $\left(\mathrm{I}_{p-p}\right)$, means and the root-mean-squares of these values for the six regions. We note that in the case of intensity, the variations in the three first frequencies (23,32 and $41 \mathrm{GHz}$ ) is even larger than the expected CMB contribution, which implies that in addition there is a significant contribution to the error budget coming from the variations of the diffuse background in the surroundings. This effect is not seen in polarization, so in this case the error bars seem to be dominated by the instrumental noise contribution. However, we note that the average values of $\mathrm{Q}_{\text {back }}$ and $\mathrm{U}_{\text {back }}$ show a small degree of diffuse polarization in the background, which is very well corrected by the ring analysis.

\subsubsection{NGC 1499: Null-hypothesis in polarization}

The California Nebula is a $\mathrm{H}$ II region close to G159.6-18.5 (its optical coordinates are RA(J2000)= $04^{h} 03^{m} 18^{s}$ and $\operatorname{Dec}=+36^{\circ} 25 \cdot 3^{\prime}$ ). Since the dominant emission mechanism in diffuse $\mathrm{H}$ II regions at these frequencies is the free-free emission, they are expected to be practically unpolarized, so NGC 1499 will be our null-hypothesis for the polarization.

California is also an extended object at this resolution. For consistency, we use the same aperture 
Table 2:: Results obtained for the six control regions in the surroundings of G159.6-18.5. Stokes I, Q, U are measured using the "ring analysis" method with an aperture of $r_{1}=2^{\circ}$ at all frequencies. Error bars in all cases include the instrumental noise contribution. In the case of Stokes I, the number in brackets indicates the expected CMB contribution to the error budget, which has to be added in quadrature to the instrumental noise. Columns 5 and 6 present the derived upper limits (at the 95\% confidence level ) on the linear polarization $\mathrm{P}$, as well as upper limit $\mathrm{P}_{0}$ that would be obtained in the null-case (i.e. using $\mathrm{Q}=\mathrm{U}=0$ ). Last two columns $\left(\mathrm{Q}_{\text {back }}\right.$ and $\left.\mathrm{U}_{\text {back }}\right)$ show the direct flux determination within the aperture $r_{1}$ without the diffuse background correction (i.e. without the subtraction of the flux in the circular corona around $r_{1}$ ).

\begin{tabular}{|c|c|c|c|c|c|c|c|}
\hline $\begin{array}{c}\text { Region A1 } \\
v[\mathrm{GHz}]\end{array}$ & $\mathrm{I}[\mathrm{Jy}]$ & $\mathrm{Q}[\mathrm{Jy}]$ & $\mathrm{U}[\mathrm{Jy}]$ & $\mathrm{P}[\mathrm{Jy}]$ & $\mathrm{P}_{0}[\mathrm{Jy}]$ & $\mathrm{Q}_{\text {back }}[\mathrm{Jy}]$ & $\mathrm{U}_{\text {back }}[\mathrm{Jy}]$ \\
\hline 23 & $-3.57 \pm 0.13( \pm 1.49)$ & 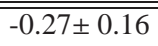 & 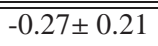 & 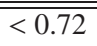 & $\overline{c<0.46}$ & $1.12 \pm 0.13$ & $-1.28 \pm 0.16$ \\
\hline 33 & $-6.05 \pm 0.23( \pm 3.00)$ & $-0.39 \pm 0.30$ & $0.43 \pm 0.38$ & $<1.48$ & $<0.82$ & $0.39 \pm 0.26$ & $-0.85 \pm 0.33$ \\
\hline 41 & $-7.71 \pm 0.32( \pm 4.59)$ & $-0.02 \pm 0.41$ & $-0.13 \pm 0.54$ & $<2.10$ & $<1.17$ & $0.02 \pm 0.37$ & $-0.86 \pm 0.49$ \\
\hline 61 & $-13.86 \pm 0.80( \pm 9.67)$ & $0.09 \pm 1.02$ & $0.16 \pm 1.31$ & $<3.29$ & $<2.86$ & $0.93 \pm 0.95$ & $-0.97 \pm 1.22$ \\
\hline 94 & $-20.81 \pm 1.92( \pm 19.98)$ & $-3.98 \pm 2.44$ & $0.95 \pm 3.19$ & $<7.26$ & $<6.95$ & $-1.00 \pm 2.29$ & $9.74 \pm 3.00$ \\
\hline
\end{tabular}

\begin{tabular}{cccccccc} 
Region A2 & \multicolumn{1}{l}{} \\
\hline \hline 23 & $3.97 \pm 0.13( \pm 1.50)$ & $0.23 \pm 0.17$ & $0.26 \pm 0.21$ & $<0.70$ & $<0.46$ & $1.75 \pm 0.13$ & $-1.30 \pm 0.17$ \\
33 & $5.91 \pm 0.23( \pm 3.02)$ & $0.01 \pm 0.30$ & $0.04 \pm 0.38$ & $<1.09$ & $<0.84$ & $0.68 \pm 0.26$ & $-1.11 \pm 0.33$ \\
41 & $7.43 \pm 0.34( \pm 4.61)$ & $-0.26 \pm 0.44$ & $0.09 \pm 0.56$ & $<1.37$ & $<1.23$ & $0.36 \pm 0.40$ & $-1.33 \pm 0.51$ \\
61 & $13.58 \pm 0.81( \pm 9.72)$ & $-0.78 \pm 1.03$ & $-0.81 \pm 1.35$ & $<3.33$ & $<2.93$ & $0.78 \pm 0.95$ & $-0.10 \pm 1.25$ \\
94 & $28.66 \pm 1.98( \pm 20.09)$ & $0.40 \pm 2.50$ & $-4.84 \pm 3.29$ & $<7.92$ & $<7.16$ & $5.48 \pm 2.34$ & $2.54 \pm 3.10$ \\
\hline
\end{tabular}

\begin{tabular}{cccccccc} 
Region A3 & $7.03 \pm 0.13$ & $-1.74 \pm 0.18$ \\
\hline \hline 23 & $6.14 \pm 0.13( \pm 1.49)$ & $-0.25 \pm 0.17$ & $-0.60 \pm 0.23$ & $<1.02$ & $<0.49$ & $1.03 \pm 0.13$ & $-0.52 \pm 0.35$ \\
33 & $5.59 \pm 0.24( \pm 2.99)$ & $-0.20 \pm 0.31$ & $0.47 \pm 0.41$ & $<1.35$ & $<0.89$ & $0.32 \pm 0.27$ & $-0.57 .07 \pm 0.54$ \\
41 & $6.40 \pm 0.36( \pm 4.57)$ & $-0.06 \pm 0.46$ & $-0.07 \pm 0.59$ & $<1.79$ & $<1.29$ & $0.42 \pm 0.41$ & $-1.07 \pm 0.39$ \\
61 & $8.86 \pm 0.89( \pm 9.63)$ & $0.48 \pm 1.13$ & $1.22 \pm 1.48$ & $<4.54$ & $<3.21$ & $1.31 \pm 1.05$ & $0.52 \pm 1.39$ \\
94 & $14.65 \pm 2.17( \pm 19.91)$ & $0.41 \pm 2.75$ & $0.22 \pm 3.59$ & $<9.90$ & $<7.81$ & $-1.84 \pm 2.59$ & $5.91 \pm 3.39$ \\
\hline
\end{tabular}

\begin{tabular}{cccccccc} 
Region A4 & \multicolumn{10}{l}{} \\
\hline \hline 23 & $-2.90 \pm 0.14( \pm 1.49)$ & $0.00 \pm 0.18$ & $-0.74 \pm 0.24$ & $<1.14$ & $<0.51$ & $1.14 \pm 0.14$ & $-1.51 \pm 0.19$ \\
33 & $-3.41 \pm 0.26( \pm 2.99)$ & $0.18 \pm 0.33$ & $-0.36 \pm 0.43$ & $<1.54$ & $<0.93$ & $0.72 \pm 0.28$ & $-0.17 \pm 0.37$ \\
41 & $-4.45 \pm 0.41( \pm 4.58)$ & $0.74 \pm 0.50$ & $1.35 \pm 0.75$ & $<2.40$ & $<1.58$ & $1.09 \pm 0.46$ & $0.79 \pm 0.68$ \\
61 & $-7.20 \pm 0.91( \pm 9.65)$ & $-0.03 \pm 1.15$ & $-2.88 \pm 1.51$ & $<4.79$ & $<3.28$ & $0.93 \pm 1.07$ & $-2.89 \pm 1.40$ \\
94 & $-11.37 \pm 2.26( \pm 19.94)$ & $1.06 \pm 2.88$ & $1.03 \pm 3.71$ & $<9.35$ & $<8.12$ & $5.22 \pm 2.71$ & $12.62 \pm 3.48$ \\
\hline
\end{tabular}

\begin{tabular}{cccccccc} 
Region A5 & \multicolumn{7}{l}{} \\
\hline \hline 23 & $-2.02 \pm 0.13( \pm 1.50)$ & $0.22 \pm 0.17$ & $-0.21 \pm 0.22$ & $<0.66$ & $<0.48$ & $1.39 \pm 0.14$ & $0.09 \pm 0.17$ \\
33 & $-1.65 \pm 0.24( \pm 3.02)$ & $0.25 \pm 0.31$ & $-0.47 \pm 0.40$ & $<0.96$ & $<0.87$ & $0.73 \pm 0.27$ & $-0.29 \pm 0.35$ \\
41 & $-1.83 \pm 0.36( \pm 4.61)$ & $0.90 \pm 0.48$ & $-0.26 \pm 0.57$ & $<2.04$ & $<1.28$ & $1.07 \pm 0.43$ & $-0.10 \pm 0.51$ \\
61 & $-2.10 \pm 0.86( \pm 9.72)$ & $-0.31 \pm 1.11$ & $0.20 \pm 1.38$ & $<4.95$ & $<3.05$ & $-0.02 \pm 1.03$ & $1.20 \pm 1.28$ \\
94 & $-11.06 \pm 2.12( \pm 20.08)$ & $1.81 \pm 2.74$ & $-2.49 \pm 3.38$ & $<8.07$ & $<7.50$ & $7.06 \pm 2.59$ & $2.43 \pm 3.19$ \\
\hline
\end{tabular}

\begin{tabular}{cccccccc} 
Region A6 & \multicolumn{7}{l}{} \\
\hline \hline 23 & $-3.95 \pm 0.13( \pm 1.49)$ & $0.15 \pm 0.17$ & $-0.13 \pm 0.22$ & $<0.57$ & $<0.48$ & $1.46 \pm 0.13$ & $-0.47 \pm 0.17$ \\
33 & $-2.91 \pm 0.24( \pm 3.00)$ & $-0.05 \pm 0.30$ & $-0.28 \pm 0.40$ & $<1.17$ & $<0.86$ & $0.80 \pm 0.26$ & $-0.39 \pm 0.34$ \\
41 & $-2.48 \pm 0.35( \pm 4.59)$ & $-0.42 \pm 0.45$ & $0.94 \pm 0.56$ & $<1.56$ & $<1.25$ & $0.65 \pm 0.40$ & $1.16 \pm 0.51$ \\
61 & $-1.34 \pm 0.83( \pm 9.68)$ & $0.17 \pm 1.06$ & $-0.09 \pm 1.37$ & $<3.44$ & $<2.99$ & $-0.26 \pm 0.98$ & $1.31 \pm 1.28$ \\
94 & $-5.80 \pm 2.04( \pm 20.00)$ & $1.73 \pm 2.59$ & $1.37 \pm 3.36$ & $<7.84$ & $<7.36$ & $4.34 \pm 2.44$ & $5.86 \pm 3.17$ \\
\hline
\end{tabular}


Table 3:: Summary of the statistics for the six control regions in table 2 We present the peak-to-peak variations ( $\mathrm{I}_{p-p}$ ), means and standard deviations of the Stokes I, Q and U, together with the mean of the $\mathrm{Q}_{\text {back }}$ and $\mathrm{U}_{\text {back }}$ values for each of the five WMAP frequencies. All quoted values are given in Jy.

\begin{tabular}{cccccccccc|cc}
\hline$v[\mathrm{GHz}]$ & $\mathrm{I}_{p-p}$ & $\langle\mathrm{I}\rangle$ & $\left\langle\mathrm{I}^{2}\right\rangle^{1 / 2}$ & $\mathrm{Q}_{p-p}$ & $\langle\mathrm{Q}\rangle$ & $\left\langle\mathrm{Q}^{2}\right\rangle^{1 / 2}$ & $\mathrm{U}_{p-p}$ & $\langle\mathrm{U}\rangle$ & $\left\langle\mathrm{U}^{2}\right\rangle^{1 / 2}$ & $\left\langle\mathrm{Q}_{\text {back }}\right\rangle[\mathrm{Jy}]$ & $\left\langle\mathrm{U}_{\text {back }}\right\rangle[\mathrm{Jy}]$ \\
\hline \hline 23 & 10.10 & -0.39 & 4.32 & 0.50 & 0.01 & 0.23 & 1.00 & -0.28 & 0.36 & 1.32 & -1.04 \\
32 & 11.96 & -0.42 & 4.99 & 0.64 & -0.03 & 0.24 & 0.95 & -0.03 & 0.41 & 0.61 & -0.55 \\
41 & 15.13 & -0.44 & 6.06 & 1.32 & 0.15 & 0.54 & 1.61 & 0.32 & 0.66 & 0.60 & -0.24 \\
61 & 27.44 & -0.34 & 10.12 & 1.26 & -0.06 & 0.44 & 4.10 & -0.37 & 1.39 & 0.61 & -0.15 \\
93 & 49.46 & -0.96 & 18.70 & 5.80 & 0.24 & 2.16 & 6.21 & -0.63 & 2.50 & 3.21 & 6.52 \\
\hline
\end{tabular}

$r_{1}=2^{\circ}$ for all frequency bands, which after visual inspection, is found to fit well with the solid angle that defines the source. The central coordinates of the emission used for the flux integration are $\mathrm{RA}=60.40^{\circ}$ and $\mathrm{Dec}=+36.3675^{\circ}$, practically coinciding with the peak emission in the $33 \mathrm{GHz}$ map. Our results on the I, Q, U measurements, and the derived upper limits on the linear polarization $4 \mathrm{P}$ and $\Pi$, as well as the nullcase $P_{0}$ and $\Pi_{0}$, are presented in table 4

A simple extrapolation of the DIRBE fluxes to the WMAP frequency range shows that the thermal dust emission is expected to be negligible between 20$100 \mathrm{GHz}$. Thus, we expect that the main emission mechanism is the free-free emission. To check this prediction, we have derived the spectral index that best-fits the data obtained in the range $23-41 \mathrm{GHz}$, using a power law model $\left(\mathrm{I} \propto v^{\beta}\right)$. We find $\beta=0.08 \pm$ 0.10 , which is apparently in contradiction with the expected frequency behaviour for an optically thin freefree emitting region at these frequencies $(\beta \approx-0.1)$. However, this seems to be a effect of the residual CMB contribution to these measurements. If we correct the WMAP maps from the CMB emission by subtracting the ILC map, the flux estimates at 23,32 and $41 \mathrm{GHz}$ are now found to be $61.6,59.6$ and $59.5 \mathrm{Jy}$, respectively. Using these values, we find $\beta=-0.07 \pm 0.11$, which is now compatible with the expected value.

Concerning the polarization measurements, at all frequencies the Stokes Q and U parameters are found to be compatible with a zero level. The upper limits on $\mathrm{P}$, and $\Pi$ at the $95 \%$ confidence level are similar to those obtained for the null-case hypothesis $\left(\mathrm{P}_{0}\right.$ and $\Pi_{0}$ ). Indeed, the full posterior distributions for these parameters are found to be very similar, implying a non-detection of polarization in the region, as one would expect for an $\mathrm{H}$ ir region.

\footnotetext{
${ }^{4} \Pi$ is the fractional linear polarization, defined as $\Pi \equiv 100 \mathrm{P} / \mathrm{I}$.
}

\subsection{Perseus}

Figure 3 shows a detailed view of the G159.6-18.5 region. The circular aperture used for the flux determination corresponds to a radius of $r_{1}=2^{\circ}$, and is centered in $\operatorname{RA}(\mathbf{J} 2000)=55.4^{\circ}$ and $\mathrm{Dec}=31.8^{\circ}$. Since G159.6-18.5 is a extended object, we have used the same aperture $r_{1}$ at all frequency bands. The subset of pixels within the small aperture at the northwest of the center of G159.6-18.5 were removed for the flux determination, as they correspond to the location of the quasar $4 \mathrm{C}+32.14$. This small aperture uses $r_{4 C+32.14}=0.25^{\circ}$ at all frequencies, and it is centered at $\mathrm{RA}(\mathrm{J} 2000)=54.125^{\circ}$ and $\mathrm{Dec}=32.308^{\circ}$. Table 5 summarizes the results obtained for the region.

\subsubsection{Intensity Signal}

The second column in Table 5 presents the results for the intensity measurements in the G159.6-18.5 region. A comparison with the results in W05 shows that our fluxes are between 5 and 13 per cent higher in the first four channels (23 to $61 \mathrm{GHz}$ ), and 10 per cent smaller in the last channel $(94 \mathrm{GHz})$. This small discrepancy is probably due to the different methodologies used to derive the fluxes. In W05, an elliptical model for the region was adopted, so any departure of the actual shape of the region from this model might introduce these changes.

Figure 4 shows our results for the intensity signal in WMAP and DIRBE maps, together with the ancillary data described in section 3.2 Following W05, using the $\mathrm{W}$ band and the DIRBE data we can fit the thermal dust emission with a modified blackbody function $\mathrm{I}_{\text {dust }} \propto v^{\beta_{\text {dust }}+2} \mathrm{~B}\left(v, \mathrm{~T}_{\text {dust }}\right)$, where $\mathrm{T}_{\text {dust }}$ is the dust temperature and $\beta_{\text {dust }}$ the dust emissivity index. The black dashed line represents the best-fit model of the thermal dust emission, which has parameters $\beta_{\text {dust }}=1.55$ and $\mathrm{T}_{d u s t}=19 \mathrm{~K}$, in full agreement with W05. These values are expected for dust in a 'warm neutral medium' 
Table 4:: Summary of flux measurements for NGC1499. We use an aperture of $r_{1}=2^{\circ}$ at all frequencies to integrate the fluxes for I, Q and U Stokes parameters. The upper limits on $\mathrm{P}$ and $\Pi$ are derived using a maximum likelihood analysis. Similarly, we get the upper limits $\mathrm{P}_{0}$ and $\Pi_{0}$ for the null-case hypothesis $(\mathrm{Q}=\mathrm{U}=0)$. As in Table 2 for the intensity column the error budget is separated in two contributions, the instrumental noise and the $\mathrm{CMB}$ error (within parenthesis).

\begin{tabular}{cccccccc}
$v[\mathrm{GHz}]$ & $\mathrm{I}[\mathrm{Jy}]$ & $\mathrm{Q}[\mathrm{Jy}]$ & $\mathrm{U}[\mathrm{Jy}]$ & $\mathrm{P}[\mathrm{Jy}]$ & $\Pi[\%]$ & $\mathrm{P}_{0}[\mathrm{Jy}]$ & $\Pi_{0}[\%]$ \\
\hline \hline 23 & $64.65 \pm 0.13( \pm 1.50)$ & $0.28 \pm 0.16$ & $-0.12 \pm 0.21$ & $<0.63$ & $<0.98$ & $<0.46$ & $<0.71$ \\
33 & $65.70 \pm 0.23( \pm 3.02)$ & $-0.22 \pm 0.30$ & $0.44 \pm 0.37$ & $<0.90$ & $<1.40$ & $<0.82$ & $<1.27$ \\
41 & $68.92 \pm 0.32( \pm 4.62)$ & $0.36 \pm 0.41$ & $0.15 \pm 0.54$ & $<1.18$ & $<1.84$ & $<1.17$ & $<1.84$ \\
61 & $70.73 \pm 0.79( \pm 9.73)$ & $0.58 \pm 1.01$ & $0.21 \pm 1.31$ & $<3.05$ & $<4.73$ & $<2.84$ & $<4.43$ \\
94 & $65.61 \pm 1.93( \pm 20.11)$ & $2.64 \pm 2.45$ & $-1.55 \pm 3.19$ & $<7.10$ & $<10.90$ & $<6.91$ & $<10.70$ \\
\hline
\end{tabular}

Table 5:: Summary of flux measurements for the G159.6-18.5 region. The meaning of the different columns is the same as in Table 4

\begin{tabular}{cccccccc}
$v[\mathrm{GHz}]$ & $\mathrm{I}[\mathrm{Jy}]$ & $\mathrm{Q}[\mathrm{Jy}]$ & $\mathrm{U}[\mathrm{Jy}]$ & $\mathrm{P}[\mathrm{Jy}]$ & $\Pi[\%]$ & $\mathrm{P}_{0}[\mathrm{Jy}]$ & $\Pi_{0}[\%]$ \\
\hline \hline 23 & $47.76 \pm 0.13( \pm 1.44)$ & $-0.07 \pm 0.16$ & $0.01 \pm 0.22$ & $<0.48$ & $<1.01$ & $<0.47$ & $<0.98$ \\
33 & $42.97 \pm 0.23( \pm 2.90)$ & $0.05 \pm 0.29$ & $0.43 \pm 0.39$ & $<0.86$ & $<1.79$ & $<0.85$ & $<1.76$ \\
41 & $37.88 \pm 0.33( \pm 4.44)$ & $-0.09 \pm 0.42$ & $-0.41 \pm 0.55$ & $<1.29$ & $<2.69$ & $<1.19$ & $<2.48$ \\
61 & $36.36 \pm 0.80( \pm 9.35)$ & $0.06 \pm 1.01$ & $0.67 \pm 1.33$ & $<3.44$ & $<7.23$ & $<2.88$ & $<6.03$ \\
94 & $70.18 \pm 1.94( \pm 19.32)$ & $1.13 \pm 2.46$ & $-2.06 \pm 3.24$ & $<7.52$ & $<15.64$ & $<7.04$ & $<14.65$ \\
\hline
\end{tabular}

WNM).

The low frequency behaviour is fitted by the dotted line to a free-free emission, using the measurement at $1420 \mathrm{MHz}(7.3 \pm 2.0 \mathrm{Jy})$ and the typical free-free spectral index $\beta_{f f}=-0.12$ for an optically thin region.

As in W05, the extrapolations of the thermal dust and free-free emissions can not explain the rising spectrum between 10 and $23 \mathrm{GHz}$, and a third component has to bee included in the fit. Following W05, we consider a model based on a linear combination of the Draine \& Lazarian (1998) models for Warm Neutral Medium (WNM) and Molecular Cloud (MC). Using $\tau_{3000}=8.45 \times 10^{-4}$ (obtained from the fit thermal dust emission) and the canonical factor of $2.13 \times 10^{24} \mathrm{H} \mathrm{cm}^{-2}=1 \tau_{100}$ (from Finkbeiner et al. 2004), we obtain the column number density of hydrogen atoms $N(\mathrm{H})=1.8 \times 10^{21} \mathrm{~cm}^{-2}$, which differs from the value obtained by W05 $\left(1.3 \times 10^{22} \mathrm{~cm}^{-2}\right)$ due to the dependence of $N(\mathrm{H})$ on the solid angle. Finally, considering our $N(\mathrm{H})$ value and using the solid angle $\Omega_{s}$ that defines the source, we find that the best-fit to the observed data is obtained with the combination $\mathrm{I}_{a} \approx 0.85 \mathrm{WNM}+0.35 \mathrm{MC}$ (black dot-dashed line in Figure 47). The red solid line in Fig. 4 corresponds to the co-added spectral energy distribution model $\mathrm{I}_{t o t}$ for the three components $\left(\mathrm{I}_{t o t}=\mathrm{I}_{a}+\mathrm{I}_{d u s t}+\mathrm{I}_{f f}\right)$.

\subsubsection{Polarization Signal}

Table 5 summarizes our polarization measurements, which show that the polarized intensity in G159.6-18.5 is compatible with zero at the five frequency bands. Because of this reason, all quoted values in columns 5 to 8 correspond to upper limits at the 95 per cent confidence level. Nevertheless, these upper limits on the linear polarization fraction still provide extremely important constraints on the nature of the anomalous emission.

Figure 5 represents our upper limits from WMAP data, together with the $11 \mathrm{GHz}$ result of Battistelli et al. (2006). For comparison, we also include the expected dust fractional polarization for three cases, namely $\Pi_{\text {dust }}$ equal to $6.0 \%$ (solid black line), $3.6 \%$ (solid blue line) and $2.0 \%$ (solid red line). This range of values for the polarized dust emission in the region are taken to be consistent with the estimates in Kogut et al. (2007). The conclusion is that our upper limits on the fractional linear polarization in G159.6-18.5 at these microwave wavelengths can be directly translated into upper limits on the anomalous emission, at least in the frequency range $23-41 \mathrm{GHz}$, while the dust contribution has to be taken into account at higher frequencies. Thus, the upper limits at 23 and $32 \mathrm{GHz}$ of $\Pi(23 \mathrm{GHz})<1.0 \%$ and $\Pi(32 \mathrm{GHz})<1.8 \%(95 \%$ C.L.) constitute the most stringent constraints on the 


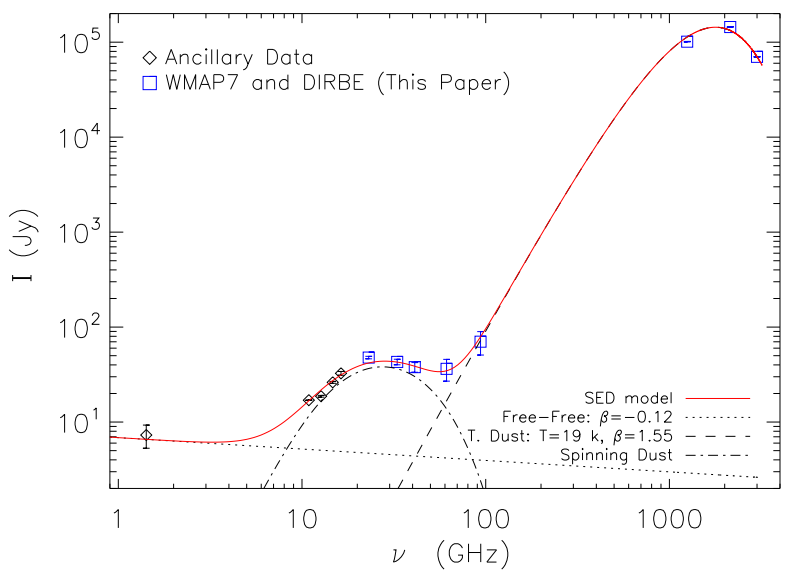

Fig. 4.-: Spectral Energy Distribution (SED) for the G159.6-18.5 region. The blue squares and black diamond correspond to measurements obtained in this paper (WMAP and DIRBE bands) and the ancillary data used (COSMOSOMAS and HASLAM bands), respectively. The SED is fitted with three contributions: i) the free-free emission $\left(\mathrm{I}_{f f}\right)$, modelled as a power law with spectral index $\beta_{f f}=-0.12$; ii) the thermal dust emission, modelled as a modified black body ( $\mathrm{I}_{\text {dust }}$ ) with $\beta_{\text {dust }}=1.55$ and $\mathrm{T}_{\text {dust }}=19 \mathrm{~K}$; and iii) the spinning dust due to the electric dipole, modelled as the linear combination of two Draine \& Lazarian (1998) models for warm neutral medium and molecular cloud $(0.85 W N M+$ $0.35 M C)$. The red line represents the co-added SED model of the three contributions. polarization of this emission.

Faraday screen model. As mentioned in Sect. 2, Reich \& Reich (2009) have recently suggested that G159.6-18.5 is acting as a Faraday screen (FS) hosting a strong regular magnetic field, which would rotate the background polarized emission, being the responsible mechanism of the signal detected in Battistelli et al. (2006). However, this mechanism produces negligible effects on the expected polarized emission at the WMAP frequencies. To show this, we can use the estimates of the background emission from the last two columns in table 3 , and take the rotation measure $R M=190 \mathrm{rad} \mathrm{m}^{-2}$ as a face value to evaluate the effect. Being conservative, we can assume that the full background emission is rotated by the FS. In that case, for the relevant rotation angles (computed as $\theta=R M \lambda^{2}$ ), we obtain that the expected polarization signal is smaller than $0.1 \mathrm{Jy}$ (or $0.2 \%$ ) at $23 \mathrm{GHz}$, and becomes even smaller at higher frequencies (Ka, Q and V). Therefore, we can safely neglect its contribution at the WMAP frequency bands.

Implications on the anomalous emission models. Figure 6 illustrates the implications of our measurements on the existing models of anomalous emission. The figure also includes the value of Battistelli et al. (2006), because even in the case that this result only provide an upper limit to the polarization of the anomalous emission, it still is a very valuable constraint.

In Figure 6 we have considered two families of the so-called spinning dust models: i) the electric dipole emission and the resonance relaxation (hereafter ED) proposed by Lazarian \& Draine (2000); and ii) the magnetic dipole emission (hereafter MD) proposed in Draine \& Lazarian (1999). For this second case, the number of variants is very large, so here we will restrict ourselves to those models where the grains that dominate the 10-100 GHz emission consist of a single magnetic domain. These models were computed by Draine \& Lazarian (1999) for metallic Fe, and for an hypothetical material X4 (defined by them). Both results will be used here, for three different grain shapes, namely $1: 1.25: 1.5$ (dotted lines), $1: 1.5: 1.5$ (dashed lines) and $1: 2: 2$ (dot-and-dashed lines). For further details on these models, see Draine \& Lazarian (1999).

We note that in addition to the anomalous emission, 


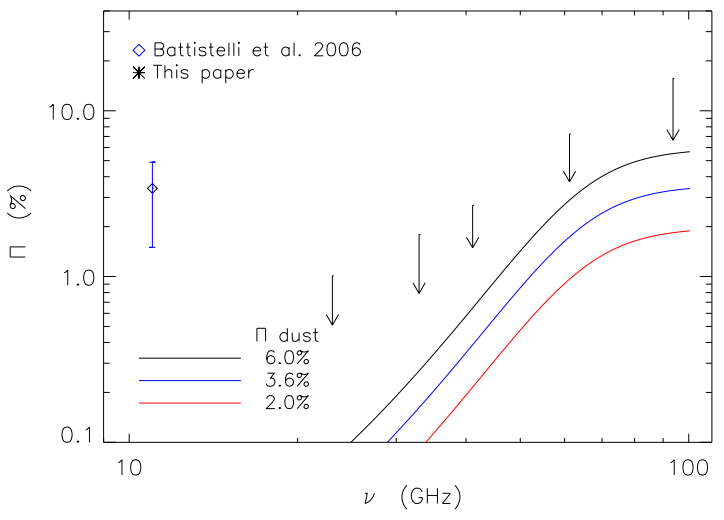

Fig. 5.- : Our measurements on the total fractional linear polarization $\Pi$ (black arrows) in the G159.6-18.5 region. We also include the result of $\Pi=3.4_{-1.4}^{+1.8} \%$ at $11 \mathrm{GHz}$ from Battistelli et al. (2006). For illustration, the solid lines show the predicted fractional linear polarization due to thermal dust in the region for three values of $\Pi_{\text {dust }}$, namely $6.0 \%$ (black line), 3.6\% (blue line) and 2.0\% (red line), which are taken to be consistent with range of values allowed by Kogut et al. (2007). At the frequencies around the peak of the anomalous emission, the constraints are of the order of 1 per cent, and can be directly translated into constraints on the polarization of the anomalous emission. when plotting the different models in figure 6 we have also included the contribution of the polarized thermal dust emission, using the value $\Pi_{\text {dust }}=6.0 \%$. In practise, this is done as described by this equation

$$
\Pi_{m}=\frac{\mathrm{P}_{a}+\mathrm{P}_{d u s t}}{\mathrm{I}_{a}+\mathrm{I}_{d u s t}+\mathrm{I}_{f f}}
$$

where the model for the linear polarization of anomalous and the thermal dust emission can be represented by $\mathrm{P}_{a}=\mathrm{I}_{a} \Pi_{a}$ and $\mathrm{P}_{\text {dust }}=\mathrm{I}_{\text {dust }} \Pi_{\text {dust }}$ respectively. The $\mathrm{I}_{d u s t}, \mathrm{I}_{f f}$ and $\mathrm{I}_{d u s t}$ contributions were obtained in the previous section.

From Figure 6 we see that the measurement at $11 \mathrm{GHz}$ can not completely rule out the model of dust grains of X4 with semiaxes 1:1.5:1.5. However, when including the upper limits derived here from WMAP data, all the considered models based on magnetic dipole emission can be ruled out. In contrast, all these measurements are in agreement with the predictions of the polarization fraction from the electric dipole and resonance relaxation theory (Lazarian \& Draine 2000) at this frequency range (see Fig. 7).

\section{Conclusions}

A detailed understanding of the physical mechanism responsible for the anomalous microwave emission is still needed. The polarization properties of those regions with anomalous emission, as G159.618.5 , are very useful tools to disentangle among the different models proposed in the literature.

Here, we used the WMAP7 data to study the intensity and polarization properties of the emission in the G159.6-18.5 region. In intensity, our results confirm the presence of the anomalous microwave emission. We present an updated SED model that takes into account the contribution of the electric dipole emission of very small dust grains rapidly rotating (Draine \& Lazarian 1998).

Concerning the polarization in the region, we present the first constraints on the polarization properties of the anomalous microwave emission at high frequencies $(23-94 \mathrm{GHz})$. Due to the fact that more of the 90 per cent of the emission in G159.6-18.5 is diffuse and extended over scales larger than 40 arcmin (Tibbs et al. 2010), the angular resolution of WMAP is not a limitation when constraining the polarization degree of the emission in the region. Although we find no detection of polarization in any of the five frequency bands, the derived upper lim- 


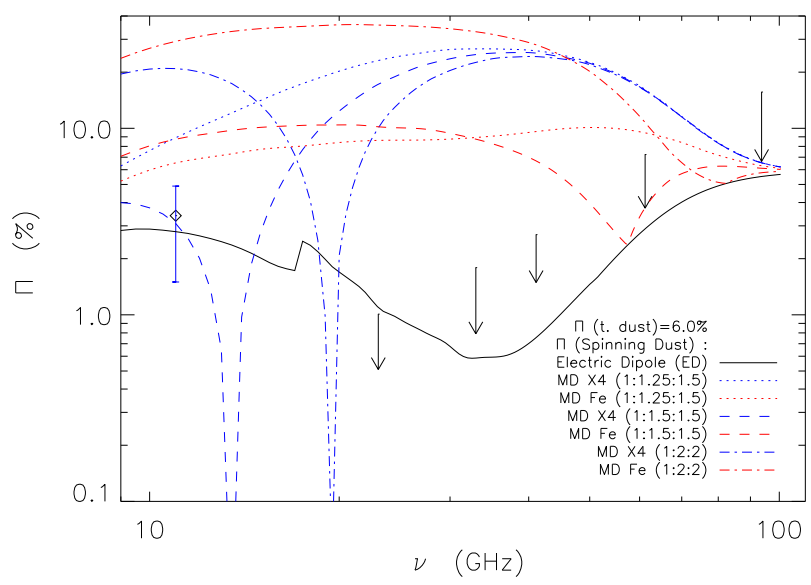

Fig. 6.- : Constraints on several anomalous emission models based on our results in the G159.6-18.5 region. Data points and upper limits have the same meaning as in Fig.5 The lines correspond to the fractional linear polarization for different spinning dust models: i) the electric dipole from Lazarian \& Draine (2000) (black solid line); and ii) the magnetic dipole emission from (Draine \& Lazarian 1999), that predicts a different frequency behaviour (blue and red lines) depending on the composition and shape of the grains. For completeness, we have added to all models the contribution of polarized thermal dust emission with a polarization fraction of $\Pi_{\text {dust }}=6.0 \%$. Our results exclude the magnetic dipole emission models as the physical process responsible of the observed polarization in G159.6-18.5 . In contrast, these are consistent with the expected linear polarization from the electric dipole emission and resonance relaxation. its allow to exclude a significant number of models based on the magnetic dipole emission of dust grains (Draine \& Lazarian 1999) as the physical process responsible of the observed polarization.

The main conclusion is that the combination of our constraints in the range 23-94 GHz, with the the measurement at $11 \mathrm{GHz}$ from the COSMOSOMAS experiment (Battistelli et al. 2006) are consistent with the expected linear polarization arising from the electric dipole model with resonance relaxation.

Further observations with higher sensitivity and angular resolution in this and other regions will be valuable to understand the nature of this anomalous emission in polarization.

We acknowledge the use of the Legacy Archive for Microwave Background Data Analysis (LAMBDA). Support for LAMBDA is provided by the NASA Office of Space Science. Some of the results in this paper have been derived using the HEALPIx (Górski et al. 2005) package. This work has been partially funded by project AYA2007-68058-C03-01 of the Spanish Ministry of Science and Innovation (MICINN). JAR-M is a Ramón y Cajal fellow of the Spanish Ministry of Science and Innovation (MICINN).

\section{REFERENCES}

Ali-Haïmoud, Y., Hirata, C. M., \& Dickinson, C. 2009, MNRAS, 395, 1055

Andersson, B., Wannier, P. G., Moriarty-Schieven, G. H., \& Bakker, E. J. 2000, AJ, 119, 1325

Banday, A. J., Gorski, K. M., Bennett, C. L., Hinshaw, G., Kogut, A., \& Smoot, G. F. 1996, ApJ, $468, \mathrm{~L} 85+$

Battistelli, E. S., Rebolo, R., Rubiño-Martín, J. A., Hildebrandt, S. R., Watson, R. A., Gutiérrez, C., \& Hoyland, R. J. 2006, ApJ, 645, L141

Bennett, C. L., Hinshaw, G., Banday, A., Kogut, A., Wright, E. L., Loewenstein, K., \& Cheng, E. S. 1993, ApJ, 414, L77

Bennett, C. L., et al. 2003, ApJS, 148, 1

Casassus, S., Cabrera, G. F., Förster, F., Pearson, T. J., Readhead, A. C. S., \& Dickinson, C. 2006, ApJ, 639,951 
Cernicharo, J., Bachiller, R., \& Duvert, G. 1985, A\&A, 149, 273

de Oliveira-Costa, A., Devlin, M. J., Herbig, T., Miller, A. D., Netterfield, C. B., Page, L. A., \& Tegmark, M. 1998, ApJ, 509, L77

de Oliveira-Costa, A., Kogut, A., Devlin, M. J., Netterfield, C. B., Page, L. A., \& Wollack, E. J. 1997, ApJ, 482, L17+

de Oliveira-Costa, A., Tegmark, M., Davies, R. D., Gutiérrez, C. M., Lasenby, A. N., Rebolo, R., \& Watson, R. A. 2004, ApJ, 606, L89

de Oliveira-Costa, A., Tegmark, M., Gutierrez, C. M., Jones, A. W., Davies, R. D., Lasenby, A. N., Rebolo, R., \& Watson, R. A. 1999, ApJ, 527, L9

de Zeeuw, P. T., Hoogerwerf, R., de Bruijne, J. H. J., Brown, A. G. A., \& Blaauw, A. 1999, AJ, 117, 354

Dickinson, C., Davies, R. D., Bronfman, L., Casassus, S., Davis, R. J., Pearson, T. J., Readhead, A. C. S., \& Wilkinson, P. N. 2007, MNRAS, 379, 297

Draine, B. T., \& Lazarian, A. 1998, ApJ, 508, 157

—. 1999, ApJ, 512, 740

Fernández-Cerezo, S., et al. 2006, MNRAS, 370, 15

Fiedler, R., Pauls, T., Johnston, K. J., \& Dennison, B. 1994, ApJ, 430, 595

Finkbeiner, D. P., Langston, G. I., \& Minter, A. H. 2004, ApJ, 617, 350

Gallegos, J. E., Macías-Pérez, J. F., Gutiérrez, C. M., Rebolo, R., Watson, R. A., Hoyland, R. J., \& Fernández-Cerezo, S. 2001, MNRAS, 327, 1178

Gold, B., et al. 2010, ArXiv e-prints

Górski, K. M., Hivon, E., Banday, A. J., Wandelt, B. D., Hansen, F. K., Reinecke, M., \& Bartelmann, M. 2005, ApJ, 622, 759

Haslam, C. G. T., Salter, C. J., Stoffel, H., \& Wilson, W. E. 1982, A\&AS, 47, 1

Hernández-Monteagudo, C., \& Rubiño-Martín, J. A. 2004, MNRAS, 347, 403

Hildebrandt, S. R., Rebolo, R., Rubiño-Martín, J. A., Watson, R. A., Gutiérrez, C. M., Hoyland, R. J., \& Battistelli, E. S. 2007, MNRAS, 382, 594
Iglesias-Groth, S. 2006, MNRAS, 368, 1925

Jarosik, N., et al. 2007, ApJS, 170, 263

—. 2010, ArXiv e-prints

Kogut, A., \& Hinshaw, G. 1996, ApJ, 464, L39+

Kogut, A., et al. 2003, ApJS, 148, 161

—. 2007, ApJ, 665, 355

Lazarian, A., \& Draine, B. T. 2000, ApJ, 536, L15

Leitch, E. M., Readhead, A. C. S., Pearson, T. J., \& Myers, S. T. 1997, ApJ, 486, L23+

Mason, B. S., Robishaw, T., Heiles, C., Finkbeiner, D., \& Dickinson, C. 2009, ApJ, 697, 1187

Miville-Deschênes, M., \& Lagache, G. 2005, ApJS, 157,302

Page, L., et al. 2007, ApJS, 170, 335

Pauls, T., \& Schwartz, P. R. 1989, in Lecture Notes in Physics, Berlin Springer Verlag, Vol. 331, The Physics and Chemistry of Interstellar Molecular Clouds - mm and Sub-mm Observations in Astrophysics, ed. G. Winnewisser \& J. T. Armstrong, $225-+$

Reich, P., \& Reich, W. 1988, A\&AS, 74, 7

Reich, W., \& Reich, P. 2009, in IAU Symposium, Vol. 259, IAU Symposium, 603-612

Ridge, N. A., Schnee, S. L., Goodman, A. A., \& Foster, J. B. 2006, ApJ, 643, 932

Scaife, A., et al. 2007, MNRAS, 377, L69

Semenova, T. A., Pariiskii, Y. N., \& Bursov, N. N. 2009, Astronomy Reports, 53, 1

Silsbee, K., Ali-Haimoud, Y., \& Hirata, C. M. 2010, ArXiv e-prints

Silverberg, R. F., Hauser, M. G., Boggess, N. W., Kelsall, T. J., Moseley, S. H., \& Murdock, T. L. 1993, in Presented at the Society of Photo-Optical Instrumentation Engineers (SPIE) Conference, Vol. 2019, Society of Photo-Optical Instrumentation Engineers (SPIE) Conference Series, ed. M. S. Scholl, 180-189

Tibbs, C. T., et al. 2010, MNRAS, 402, 1969 
Watson, R. A., Rebolo, R., Rubiño-Martín, J. A., Hildebrandt, S., Gutiérrez, C. M., FernándezCerezo, S., Hoyland, R. J., \& Battistelli, E. S. 2005, ApJ, 624, L89
This 2-column preprint was prepared with the AAS $\mathrm{L}_{\mathrm{E}} \mathrm{X}$ macros v5.2.

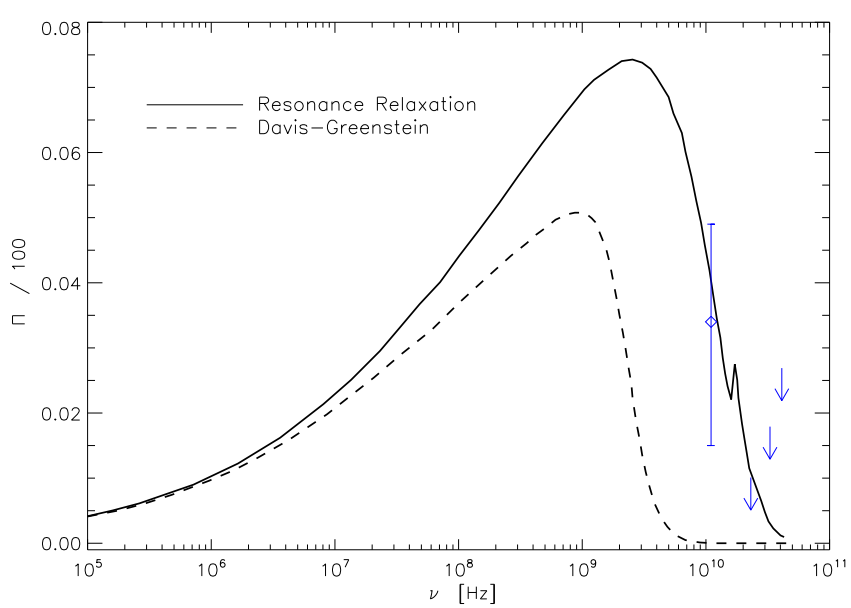

Fig. 7.-: Constraints on the grain alignment for both resonance and Davis-Greenstein relaxation models for grains in the cold interstellar medium as a function of the frequency. Both theoretical curves are taken from Lazarian \& Draine (2000). For the resonance relaxation the saturation effects are neglected, which means that the upper curves correspond to the maximal values allowed by the paramagnetic mechanism. The data point corresponds to the Battistelli et al. (2006) result, while the three upper limits are the results in this paper. 